\title{
Psychogenic paralysis: A neuroanatomical explanation of conversion
}

\section{Jordan W. Squair}

\section{University of British Columbia}

Patients who enter a neurological clinic presenting with extreme, unexplained motor weakness or paralysis without any other observable neurophysiological deficits are often diagnosed with a rarely discussed disorder: psychogenic paralysis. ${ }^{1}$ This disorder is extremely debilitating, poorly understood, ${ }^{2}$ and treatment options are currently limited. ${ }^{3}$ Recently Hallett ${ }^{3}$ discussed the disturbing reality of the situation. Not only are there an increased number of patients presenting with these unexplained symptoms ( 5 per $100,000$ people, with an average age of 30$)^{1}$ but physicians are currently unclear how to treat them. Furthermore, the prognosis for these patients is extremely negative. ${ }^{3}$ Shifting our perspective and providing a multidisciplinary approach may be necessary for developing new treatments and improving patient care.

Although Freud referred to psychogenic paralysis as a "conversion disorder", he was among the first to suggest an explanation for these patients' symptoms. ${ }^{1}$ He proposed these unexplained motor symptoms were a result of internal mental conflict, and that the expression of the symptoms helped to partially resolve this internal conflict, termed primary gain. ${ }^{1}$ Furthermore, the expression of the symptoms rewards the individual with a "sick" status, and offers them benefits such as increased attention or time away from employment, termed secondary gain. ${ }^{1}$ Therefore, the patient's internal emotional conflict may be altering their motor function while motivating them to seek any benefits that may be associated with their disability. Although Freud may be partially correct in his theory, it does not provide a framework to begin understanding the underlying neurophysiological mechanisms. Thus, a movement to bridge psychodynamics and neurophysiology is crucial to furthering our understanding of psychogenic paralysis and to improve the prognosis for these patients .,5
With advancements in imaging techniques, specifically functional magnetic resonance imaging (fMRI), we may be able to begin approaching psychogenic paralysis from a more neurophysiological point of view. ${ }^{3} \mathrm{fMRI}$ studies reveal that psychogenic paralysis patients consistently show drastic over-activation of the amygdala and associated limbic regions, ${ }^{6}$ coupled with an inhibition of sensorimotor areas. ${ }^{7}$ These limbic regions, and more specifically, the amygdala, have been implicated in a variety of emotional and motivational functions; ${ }^{8}$ thus, these results suggest emotional areas of the brain may be responsible for the inhibition of motor areas, resulting in motor weakness or paralysis. Exploring the neuroanatomy of these regions reveals that they are heavily connected through parallel basal ganglia circuits and common mediation points such as the thalamus ${ }^{9}$ and frontal lobes. ${ }^{6}$ Therefore, it is highly likely that the limbic areas and motor areas modulate each other through their common structures. Researchers have begun applying this knowledge to treatment techniques by seeking to enhance activation levels in motor areas using brain stimulation. ${ }^{1}$ Patients appeared to show improvements in motor abilities; however, more work is needed to rule out alternative explanations. ${ }^{1}$

fMRI data and an understanding of the underlying neuroanatomy provide a neurophysiological mechanism by which patients may be experiencing motor weakness. However, as important as these findings are, they do not discount Freud's original interpretation of psychogenic paralysis. Freud was convinced internal conflict ultimately led to the appearance of motor deficit. ${ }^{1}$ Based on our current understanding of psychogenic paralysis, Freud may not have been so wrong. A dramatic internal conflict would undoubtedly result in increased amygdala function. The real question is: if the conflict is so severe, can 
C this over-activation become so great as to shut down, or inhibit, other areas of the brain? Based on current theories of psychogenic paralysis, and fMRI evidence, the answer may be yes.

The bridge between psychodynamics and neurophysiology does not stop there. Freud's second postulate about psychogenic paralysis was that it provides the patient with secondary gain. ${ }^{1}$ One might characterize secondary gain as a covert motivation. Through further inspection of neuroanatomical connections one can observe that the amygdala is strongly connected to motivational centres, such as the nucleus accumbens, through the limbic channel of the basal ganglia circuit. ${ }^{10}$ Therefore, there may be a neurophysiological explanation to Freud's secondary gain concept.

Original theories surrounding psychogenic paralysis may indeed line up with neurophysiological evidence. Thus, when patients suffering from these disorders seek physicians, a multidisciplinary approach is crucial. Not only should a psychiatrist be involved to begin dealing with the underlying emotional state and its affective result, a neurologist should also be sought to examine the neurophysiological substrate of the patients' paralysis. Furthermore, as psychodynamics may provide us a more complete understanding of what the patient is experiencing, the expertise of a psychologist may also be warranted.

The junction of psychodynamics and neurophysiology is crucial for a well-rounded understanding of how psychogenic paralysis affects patients. Appreciating the neurophysiology behind Freud's primary and secondary gain concepts will give physicians better insight into the patient's psychiatric state. Only through appreciating the patient as a whole and bridging these different medical disciplines can we unlock the mysteries of these harrowing disorders.

\section{References}

1. Nowak DA, \& Fink GR. Psychogenic movement disorders: Aetiology, phenomenology, neuroanatomical correlates and therapeutic approaches. Neuroimage. 2009;47:1015-1025.

2. Ellenstein A, Kranick SM, Hallett M. An update on psychogenic movement disorders. Curr Neurol Neurosci Rep. 2011;11:296-403.

3. Hallett M. Psychogenic movement disorders: A crisis for neurology. Curr Neurol Neurosci Rep. 2006;6:269-271.

4. Kranick SM, Gorrindo T, Hallett M. Psychogenic movement disorders and motor conversion: A roadmap for collaboration between neurology and psychiatry. Psychosomatics. 2011;52:109-116.

5. Peralta V, Lang AE. Crossing the borders between neurology and psychiatry in functional neurological disorders. Mov Disord. 2011;26(8):1373-1374.

6. Voon V, Brezing C, Gallea C, Ameli R, Roelofs K, LaFrance Jr WC, et al. Emotional stimuli and motor conversion disorder. Brain. 2010;133:1526-1536.

7. Burgmer $M$, Konrad $C$, Jansen A, Kugel $H$, Sommer J, Heindel $W$, et al. Abnormal brain activation during movement observation in patients with conversion paralysis. Neuroimage. 2006;29:1336-1343.

8. Vuilleumier P, Chicherio C, Assal F, Schwartz S, Slosman D, Landis T. Functional neuroanatomical correlates of hysterical sensorimotor loss. Brain. 2001;124:1077-1090.

9. Krebs C, Weinberg J, Akesson E. Lippincott's illustrated review of neuroscience. R.A. Harvey. (Ed.). Baltimore, MD: Lippincott Williams \& Wilkins; 2011.

10. Blumenfeld $\mathrm{H}$. Neuroanatomy through clinical cases. 2nd ed. Sunderland, MA: Sinauer Associates, Inc; 2010.

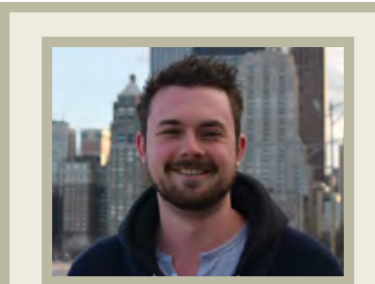

\section{Jordan Squair}

Jordan Squair is a current Master's student in the department of Kinesiology at the University of British Columbia. His research interests include the detection of preserved motor pathways in motor-complete spinal cord injured patients and how training of these pathways may improve rehabilitation outcomes. 